\title{
Mujeres en sanidad: entre la igualdad y la desigualdad
}

\author{
$M^{\mathrm{a}}$ Inmaculada PASTOR GOSÁLBEZ \\ Departamento de Gestión de Empresas \\ Instituto de Estudios del Trabajo \\ Universidad Rovira i Virgili \\ inma.pastor@urv.cat \\ Ángel BELZUNEGUI ERASO \\ Departamento de Gestión de Empresas \\ Instituto de Estudios del Trabajo \\ Universidad Rovira i Virgili \\ angel.belzunegui@urv.cat \\ Paloma PONTÓN MERINO \\ Departamento de Gestión de Empresas \\ Instituto de Estudios del Trabajo \\ Universidad Rovira i Virgili \\ paloma.ponton@urv.cat
}

Recibido: 30-09-2011

Aceptado: 29-05-2012

\begin{abstract}
This paper aims to analyze the obstacles that female doctors should fight along their career. We affirm that the process of feminization of professions like Medicine does not result in an improvement for women professional careers. We also affirm that leadership positions are not distributed proportionately between men and women. The increase of women among Medicine students, residents and doctors is not accompanied by a proportional growth of women in management and leadership positions in hospitals, but there is a low representation of women in top positions. We also point that medical practice is not unrelated to gender discrimination existing in our society but, unlike the widespread view, is a reflection
\end{abstract}

Keywords: gender, hospitals, organizations, inequalities, professional career

\section{Women in the health profession: between equality and inequalities}

\section{RESUMEN}

Este artículo da cuenta de los obstáculos que las mujeres médicas deben combatir a lo largo de su trayectoria profesional. Mantenemos que los procesos de feminización de profesiones como la medicina no están dando como resultado una mejora en las trayectorias profesionales de las mujeres, en relación con su formación académica, y que los puestos de responsabilidad no están distribuidos de manera proporcional entre hombres y mujeres. El aumento de las estudiantes de medicina, de las residentes y de las médicas facultativas no está acompañado de un crecimiento proporcional de las profesionales en puestos de gestión y dirección en los hospitales, sino que existe una escasa representación de las mujeres en los altos cargos. 
Además se muestra que la práctica médica no es ajena a la discriminación por razón de género existente a la sociedad, sino que contrariamente a la visión generalizada, es un reflejo.

Palabras clave: género, hospitales, organizaciones, desigualdades, trayectorias profesionales

\section{REFERENCIA NORMALIZADA}

Pastor, I.; Belzunegui, A.; Pontón, P. (2012). Mujeres en sanidad: entre la igualdad y la desigualdad. Cuadernos de Relaciones Laborales. Vol. 30, núm. 2, p. 497-518.

SUMARIO: Introducción. 1. Algunas explicaciones teóricas para las diferencias de género en las trayectorias profesionales de médicos y médicas. 1.1. Mujer, poder y toma de decisiones. 1.2. La construcción de la identidad y la adjudicación de roles dentro de las instituciones. 1.3. La fuerza de la socialización histórica en la medicina. 1.4. Las dinámicas dentro de las instituciones sanitarias como limitación a la promoción. 2. Caracterizando a los médicos y a las médicas: entre las diferencias y las desigualdades. 3. Discursos que legitiman: lo que dicen las entrevistas. 3.1. Sobre las formas de inserción, de promoción y los perfiles profesionales. 3.2. Sobre la naturalización y la interiorización de las características sociales del género. 3.3. Sobre el capital social y las dinámicas intra institucionales. 4. Conclusiones. 5. Referencias bibliográficas.

\section{Introducción}

En los últimos años la profesión médica ha experimentado un importante proceso de feminización. Este proceso se constata ya que ha crecido tanto el número de mujeres que estudian esta profesión como el de las que la ejercen. De 9.061 estudiantes de Ciencias de la Salud que en el curso 2008-2009 había en las universidades catalanas, el $74 \%$ eran mujeres (un total de 6.702). En el conjunto de España, para el curso 2007-08 hubo 57.574 matriculados en estudios de Ciencias de la Salud, de los cuales un $71 \%$ fueron mujeres (un total de 41.041). Igualmente, ha crecido la incorporación de la mujer a la profesión médica: en 1985, en España, las mujeres representaban el $25 \%$ del conjunto de médicos, mientras en 2006 representaron el $43 \%{ }^{1}$. Para Cataluña, el total de médicos colegiados en 2009 fue de 36.645 , de los cuales, las mujeres representan un 47\% (Camps, 2009). En concreto, según Arrizabalaga y Valls-Llobet (2005), el 75\% de los médicos de nueva incorporación son mujeres, lo que conlleva una edad media de las mujeres médicas inferior a la de los varones médicos, 42 años frente a 50 años, respectivamente. El incremento de mujeres con la titulación en medicina y el aumento de mujeres en el ejercicio de esta profesión son una realidad constatada que permite hablar de la incorporación de las mujeres también a esta actividad laboral. Es un hecho que se puede interpretar como una mejora en las desigualdades laborales tradicionales entre hombres y mujeres. Sin embargo, son diversos los estudios del mercado de trabajo que constatan que el acceso masivo de las mujeres al mercado de trabajo se ha llevado a cabo

${ }^{1}$ En el mismo sentido ilustran los datos relativos a Andalucía ya que en el 2006 del $100 \%$ de personal que ingreso al Servicio Andaluz de la Salud, el 86,6\% eran mujeres. 
al mismo tiempo que se generaban nuevas desigualdades (Torns, 1999; Maruani et al, 2000).

Las nuevas desigualdades vienen caracterizadas por diversos factores entre los que destacan una segregación tanto horizontal como vertical de la ocupación laboral femenina (Torns, 2007). Ante esta realidad se puede plantear un interrogante: iel incremento de mujeres con la titulación en medicina y el aumento de mujeres en el ejercicio de esta profesión (cambios éstos que afectan a las pautas de acceso al trabajo remunerado por parte de las mujeres) están suponiendo la desaparición de las discriminaciones por razón de género o, más bien, los procesos de cambios van en dirección de transformar las discriminaciones existentes? ¿Cuáles serían estas nuevas desigualdades?

Este artículo presenta un estado de la cuestión acerca de los obstáculos que las mujeres profesionales de la medicina deben combatir a lo largo de su trayectoria profesional, tomando como apoyo empírico los resultados de una investigación ${ }^{2}$ centrada en este sector de actividad.

Partimos de la consideración, siguiendo el análisis de Torns (2007), que las discriminaciones por razón de género tradicionalmente existentes en el mercado de trabajo están experimentando un proceso de transformación (Maruani et al, 2000) y que este proceso se da, principalmente, en la dirección de disminución de las discriminaciones directas mientras se consolidan las discriminaciones indirectas (techo de cristal, discriminación salarial, acoso sexual...). En concreto, mantenemos que los procesos de feminización de determinadas profesiones no implican necesariamente una mejora de las trayectorias profesionales de las mujeres en relación con su formación académica; y que los puestos de responsabilidad (también en sectores muy feminizados en su base, como el sanitario) no están distribuidos de manera proporcional entre hombres y mujeres, sino que la presencia de ellos sigue siendo mayoritaria en estos puestos.

En definitiva, el objetivo principal de este artículo es analizar las barreras internas y externas que obstaculizan el acceso de las mujeres a los puestos de alta responsabilidad, en las organizaciones sanitarias. Creemos que se necesita más información sobre el desarrollo de las carreras profesionales diferenciales de hombres y mujeres ya que, como han puesto de manifiesto diversas especialistas, la segregación ocupacional vertical es el verdadero núcleo duro de las desigualdades de género (Nicolson, 1997; Torns, 1999; Maruani, 2000; Ely y Meyerson, 2000; Ely y Padavic, 2007).

${ }^{2}$ Investigación del Plan Nacional I+D+I, financiada por el Instituto de la Mujer (20062008), "Mujeres en la sanidad: trayectorias profesionales y acceso a cargos de responsabilidad". Número de expediente: 48/05. 


\section{Algunas explicaciones teóricas para las diferencias de género en las trayectorias profesionales de médicos y médicas}

\subsection{Mujer, poder y toma de decisiones}

La feminización del sector médico es un fenómeno social que ha aumentado en los últimos años en España. De hecho, el porcentaje de mujeres en la profesión médica supera al porcentaje de mujeres en el empleo total en un $15 \%$ (los datos generales de empleo, según la EPA de 2006 sitúan a las mujeres ocupadas en un 40,5\%). Los estudios demográficos sobre el sector médico coinciden en que la medicina se feminiza a gran velocidad. En asistencia primaria ya hay más mujeres que hombres, y en la medicina especializada superan el $40 \%$. Pero el aumento de las estudiantes de medicina, de las residentes y de las médicas facultativas no está acompañado de un crecimiento proporcional de las profesionales en puestos de gestión y dirección en los hospitales. Existe una escasa representación de las mujeres tanto en los altos cargos profesionales, académicos y sindicales, así como en las especialidades de mayor autoridad (Ulstad, 1993; Milles, 1991; Ortiz Gómez et al., 2003), fenómeno que no es exclusivo del sector sanitario, y bastamente tratado en la literatura.

El informe de 2009 sobre "Las mujeres y la toma de decisión" (THEMIS, 2009) señala que los puestos de trabajo relacionados con la administración del Estado están ocupados mayoritariamente por hombres $(79,2 \%)$. En 2010, las mujeres representaban el 32'9\% de los altos cargos de la Administración General del Estado (INE, 2010). Incluso en el caso del Poder Judicial, donde la presencia femenina es mayor $(47 \%$ de mujeres según INE, 2010) la mayoría de los magistrados de los órganos más importantes son hombres: Tribunal Supremo, 91\%; Tribunal Constitucional, 83\%; Consejo General del Poder Judicial, 67\%; Consejo Fiscal, 69\%; Tribunales Superiores de Justicia autonómicos, 94\%.

El Informe Anual sobre Desarrollo Humano (2006) de la ONU propone el índice de potenciación de género (IPG), que mide la desigualdad de género en esferas clave de la participación económica y política y de la adopción de decisiones. Los resultados son esperanzadores para las mujeres españolas: mientras que en el año $1996 \mathrm{el}$ IPG era de 0,49; en 2005 sube a 0,75. La Encuesta de Población Activa (EPA) del INE también confirma esta tendencia a acortar distancias entre hombres y mujeres en puestos de dirección y responsabilidad. No obstante, esta tendencia positiva no es igual en los diferentes ámbitos: el crecimiento de la participación de la mujer en cargos directivos y de gestión en las instituciones sanitarias parece haber crecido poco, a pesar de la feminización de la profesión médica (Arrizabalaga y VallsLlobet, 2005; Reed et al., 2001, Reed, 2001).

Ortiz Gómez et al. (2004) ponen en evidencia que siguen existiendo desigualdades que se manifiestan en procesos de segregación vertical y dificultades para la participación de las mujeres en puestos de responsabilidad, poder y autoridad profesional y científica. En otro artículo, Ortiz Gómez (2007) concluye que el género es un determinante de las oportunidades sociales, y por tanto, incide sobre las trayectorias profesionales de los hombres y las mujeres. La autora señala que es fundamen- 
tal comenzar a realizar una mirada sexuada a la práctica profesional sanitaria e identificar la presencia de hombres y mujeres, así como de los espacios que ocupan en las profesiones sanitarias.

Colomer y Peiró (2002) observaron las diferencias de género dentro de la Sociedad Española de Salud Pública y Administración Sanitaria (SESPAS). Los resultados demostraron no sólo que las mujeres participan menos de ésta Sociedad sino también de los cargos directivos (junta directiva, órganos de publicación, comités científicos, etc.). Además, las autoras mostraron que sólo con hacer visibles los resultados de su investigación se instituyó un impacto positivo en la participación femenina. Las mujeres que pudieron acceder a estos datos se organizaron y presionaron para tener presencia, tanto en órganos de poder como en las actuaciones científicas que convoca la SESPAS.

Arrizabalaga y Valls-Llobet (2005) señalan que, a pesar de la feminización del sector médico, la promoción es dispar entre hombres y mujeres. Para demostrar esto, las autoras estudiaron el acceso de las mujeres doctoras a las cátedras universitarias de medicina, a las áreas de investigación, a los puestos directivos y a los cargos de gestión de los centros sanitarios españoles. En 2001, sólo el 24\% de los puestos de atención primaria estaba ocupado por mujeres. Además se constata que sólo un 7\% de los cargos de responsabilidad en la atención especializada de los hospitales y centros de especialidades públicos de España los ostentan las mujeres. En los centros de atención primaria, donde las mujeres son amplia mayoría (el 53\% de los facultativos), ellas sólo ocupan uno de cada cuatro cargos de gerencia. Además, las mujeres docentes se promocionan menos que sus compañeros masculinos. Otro dato interesante que resulta de esta investigación es que la probabilidad de que la mujer médica sea discriminada crece en el ámbito académico. Las mujeres son amplia mayoría en las aulas de las universidades de medicina ( $72 \%$ de las matrículas). Pero, en 2011, de 27 facultades de Medicina, sólo cuatro decanatos estaban ocupados por mujeres (las decanas de la Universidad de Cádiz, Universidad Cardenal Herrera CEU de Valencia, Universidad San Pablo CEU de Madrid y Universitat Rovira i Virgili de Tarragona).

\subsection{La construcción de la identidad y la adjudicación de roles dentro de las instituciones}

Uno de los factores que explica las diferencias de género en el acceso a los puestos de responsabilidad es el rol que se le adjudica a la mujer dentro de la familia. Se repite bastante la hipótesis de la incompatibilidad entre la vida familiar y el éxito profesional. La exigencia en horas y compromiso que tienen las jefaturas de servicio, las direcciones médicas y otro tipo de cargos jerárquicos sería irreconciliable con las tareas relacionadas con el hogar y la familia.

Una encuesta realizada a pacientes pone en evidencia que existe una percepción generalizada acerca del rol social que debe ocupar la mujer y su vida laboral. Una media del $70,5 \%$ de los entrevistados piensa que es más difícil para las mujeres ser médicas y atender las tareas domésticas. Este porcentaje aumenta entre aquellos que tienen más de 45 años $(77,20 \%)$ (Cuesta Rubio et al., 2006). Otros estudios han 
demostrado que los pacientes asocian la excelencia profesional a los hombres y coinciden en que, en cambio, las mujeres son mejores escuchando y poseen mayor empatía y humanidad (Fennema et al., 1990; Delgado Sánchez et al., 1999).

Este discurso social hegemónico se define por asociar el sexo con unas determinadas características más allá de lo profesional, relacionadas con un estereotipo de las mujeres (cuidadoras, sensibles, familiares, menos fuertes, menos tecnificadas). $\mathrm{Y}$ este estereotipo está presente tanto entre los pacientes como entre los profesionales del sector sanitario. El impacto de esta construcción social de significados y sentidos en torno a la diferencia sexual es una de las hipótesis causales que explican la desigualdad de la participación de las médicas en cargos de gestión (Riska y Wegar, 1993). Arrizabalaga y Valls-Llobet (2005) señalan que este rol social y cultural asignado a la mujer impacta sobre su identidad y obstaculiza la incorporación de éstas a puestos de mando dentro de los hospitales. Otro estudio publicado en 1993 por la revista Annals of Internal Medicine, sigue este mismo argumento: los miembros de las familias formadas por médicos suelen representar los roles tradicionales de su sexo. El artículo señala que ellas suelen disfrutar de un menor éxito profesional, ya que desempeñan simultáneamente otras responsabilidades además de la laboral, y todas ellas tienen preferencia sobre el rol profesional.

Delgado Sánchez et al. (2003) señalan, partiendo de las teorías feministas de Jean B. Miller y Mabel Burín, que muchas mujeres asumen el discurso dominante, lo incorporan en sus discursos y lo reproducen en su práctica profesional. Miller ha señalado que la identidad de las mujeres, su bienestar y su fortalecimiento narcisista va a depender del sentimiento de formar parte de una relación, del "yo en relación" (Miller, 1987). Burín (1996) pone de manifiesto que entre las mujeres, el ideal del yo se articula con ser comprensivas, ser afectuosas, estar disponibles y ser cuidadoras. Estos valores del signo mujer se asocian, al mismo tiempo, al sentido que se adjudica al rol de las madres. Con estas herramientas conceptuales, Delgado Sánchez et al. (2003) establecen una relación entre la profesión y la construcción de la identidad femenina. "Como consecuencia de su estructura normativa (súper yo), muchas mujeres abandonan otras facetas de su vida para cuidar de sus hijos, o bien eligen una profesión que, según su criterio, armonice con el desarrollo de ese rol materno. Este tipo de elección implica una jerarquía de valores donde lo primero, casi siempre, es la atención a los otros y lo último el propio desarrollo personal. En muchos casos, además, los otros son, exclusivamente, la pareja, los hijos y la familia" (Sánchez et al., 2003; 593).

En síntesis, las médicas no se escapan de las desigualdades relacionadas con el género. No obstante, el contexto profesional crea sus propios significados y sentidos que producen en el interior del campo médico relaciones de poder entre hombres y mujeres. Así, a las mujeres médicas se les imprimen ciertos calificativos que restringen el acceso a cargos de responsabilidad. Si bien como médica puede ser una excelente profesional, como mujer está asociada al espacio privado, familiar, material. No obstante, en un estudio de Schroen se demuestra que no existe correlación significativa entre la maternidad y la vida familiar y la menor promoción de las mujeres médicas y que el peso de los estereotipos recae más sobre aquellos que 
seleccionan el personal y que esta variable pesaría más a la hora de explicar la desigualdad en el acceso (Schroen et al., 2004).

\subsection{La fuerza de la socialización histórica en la medicina}

Otra línea de estudios sobre las médicas es la histórica. Según se pone de manifiesto en diversos estudios (Cabré i Pairet et al., 2001; Dodd et al.1994; Elston, 2001; Ortiz Gómez et al., 1999; Ortiz Gómez, 2007), dentro de la historia de la medicina la mujer ha estado sometida a mecanismos de exclusión o subordinación que la han limitado a acceder a tareas y especialidades de menor importancia en materia de investigación, generación de conocimiento o toma de decisiones.

Antes de 1900 las mujeres no eran admitidas en las aulas y, aunque luego se erradicó esta prohibición, durante mucho tiempo la segregación de las mujeres a determinadas actividades de carácter marginal y subalterno fue eficaz y la discriminación continuó por otras vías. En el último tercio del siglo XX mujeres pertenecientes a la clase media comenzaron a estudiar medicina, pero la medicina se siguió desarrollando a través de identidades profesionales sexuadas (Ortiz Gómez, 1999). Cabe destacar el caso de la pionera Pilar Tauregui, que en 1881, fue de las primeras mujeres en España en acceder a la Escuela de Medicina; fue apedreada por sus compañeros hombres en clase porque consideraban que desprestigiaba a los estudios de medicina, y cuando terminó sus estudios no le dieron título sino un certificado que no le permitía ejercer la profesión (Anderson, Zinsser, 1991).

En otro texto, Ortiz Gómez (2007) señala que ha sido muy efectivo el efecto socializador que jugaron los programas de educación sanitaria (PES) para mujeres que, durante el último siglo y medio, servían para convertirlas en agentes de salud en el ámbito doméstico. En España, los PES se tradujeron en cursos, charlas, visitas o adiestramiento individualizado a través de diferentes instituciones benéficas, escuelas de maternidad y cartillas sanitarias; o asignaturas exclusivas para niñas y adolescentes que tenían como contenido la crianza, la higiene doméstica y la salud en el hogar. Estos mecanismos históricos de segregación contribuyeron a la selección de las especialidades según el sexo. Así, Cabré i Pairet y Ortiz Gómez (2001) confirman la idea de que existe una construcción androcéntrica de la medicina a través de la historia, copada por hombres y por valores masculinos.

El peso puesto en el factor histórico ha sido destacado también por estudios de otros países y constituye una corriente de estudio sobre las trayectorias médicas y las desigualdades producto del género. Elston (2001) pone el énfasis en los hospitales construidos a finales del siglo XIX y XX en Gran Bretaña administrados por médicas y dirigidos a las mujeres, bajo la creencia general que la atención médica en hospitales dirigidos por médicas era más apropiada a las necesidades de las mujeres y no para atender a todo tipo de pacientes. Este juicio era sostenido no solamente por aquellas personas que estaban relacionadas con el ámbito sanitario, sino también por las propias directoras de los denominados the women-run medical institutions. Dichos hospitales y su planta de profesionales constituían una red en la cual se superponían las amistades y los compromisos profesionales entre las organizaciones profesionales de médicos mujeres (Association of Registered Medical 
Women, Medical Women's Federation). Así, se sostenía una coordinación informal y se fue constituyendo una concepción compartida en torno a su rol maternal.

Estos análisis históricos sostienen que la inercia de una tradición en el tiempo permite entender el lugar de las mujeres en la medicina, su asignación en diferentes profesiones del campo de la salud y las relaciones de poder que se producen en este ámbito.

\subsection{Las dinámicas dentro de las instituciones sanitarias como limitación a la promoción}

Es difícil distinguir entre los diferentes obstáculos que tiene una médica para acceder a puestos de toma de decisiones, ya que las causas se funden unas con otras. La literatura pone de relieve que las mujeres tienen, en general, obstáculos para asumir responsabilidades dentro de las empresas e instituciones. Muchas veces son las mujeres mismas las que se autolimitan asumiendo un discurso que beneficia a los hombres; otras veces son otros (por ejemplo, los seleccionadores de personal) quienes ponen obstáculos a su promoción, al asociar a las médicas con ciertos estereotipos que no son compatibles con la carrera jerárquica. Finalmente, una parte de la literatura apunta que las exigencias a las que están sometidas las mujeres hacen que éstas terminen por disminuir su rendimiento $\mathrm{y}$, probablemente, su éxito en la trayectoria laboral. Todas estas variables interactúan obstaculizando la promoción jerárquica entre las médicas (Reed et al., 2001). No obstante, hay que analizar con cuidado aquella variable que está relacionada con las exigencias y con las dinámicas dentro de las instituciones sanitarias y, en particular, de los hospitales.

El techo de cristal es un concepto muy usado para hablar de las limitaciones institucionales. Burin (1996) lo define como el conjunto de acciones y prácticas basadas en redes formales e informales que excluyen a las mujeres de los cargos de mando y poder. Esto termina por interpelar a las mujeres e impacta sobre la subjetividad, aceptando su lugar dentro de las organizaciones sanitarias. Colomer y Peiró (2002) recurren a este concepto para señalar que no existe evidencia científica ni social de que las desigualdades se resuelvan espontáneamente. Según las autoras, el techo de cristal es producto de un tipo de retórica que hay que transformar, por ejemplo generando políticas para provocar roturas en el techo.

El acoso moral y sexual es una forma de discriminación que sufren las mujeres dentro de la profesión médica. Arrizabalaga y Valls-Llobet (2005) señalan que, gracias a vías judiciales, se ha conocido que el acoso moral y sexual dirigido contra las médicas es alarmante y que un $44 \%$ de las profesionales de la sanidad han sufrido discriminación laboral. Gil Deza $(2005)^{3}$ cita este fenómeno: según veinti-

${ }^{3}$ Las cifras no corresponden a España, sino que son el resultado de un metanálisis realizado a artículos obtenidos electrónicamente a partir de diferentes bases de datos. Los artículos originales donde se publicaron estos resultados son: SCHERNHAMMER, E. S.; COLDITZ, G. A. (2004). "Suicide rates among physicians: a quantitative and gender 
cinco investigaciones, existe un $40 \%$ más de suicidio entre los médicos con respecto a otros profesionales en la misma edad y un 130\% más de suicidio entre las médicas. La diferencia que desfavorece a las médicas se debe fundamentalmente a dos cosas: el acoso sexual hacia las médicas en el trabajo (hay una correlación directa entre acoso sexual e intento de suicidio) y la insatisfacción laboral por no poder acceder a puestos de dirección. Estos indicadores muestran la discriminación y las presiones en el interior de las instituciones a las cuáles se somete a las médicas por su género.

El llamado burnout (síndrome del profesional quemado), variable relacionada íntimamente con la anterior, permite explicar de manera indirecta la desigualdad en el acceso a cargos de responsabilidad dentro de los hospitales. Según Arrizabalaga y Valls-Llobet (2005) las médicas tienen mayor probabilidad de sufrir depresión, burnout y acoso moral en el trabajo que sus colegas masculinos. Las autoras manifiestan que los médicos expresan la insatisfacción a través de síntomas somáticos diversos y las médicas la manifiestan mediante el desgaste psíquico o burnout. Esta enfermedad laboral es más frecuente en el personal de los hospitales que en el de los centros de salud, siendo el perfil típico una mujer, mayor de 44 años, sin pareja estable, con más de 19 años de antigüedad profesional y más de 11 años en el mismo centro de trabajo, dedicando a los enfermos al menos el $70 \%$ de su jornada laboral y trabajando entre 36 y 40 horas semanales. Probablemente, el efecto burnout es un obstáculo para que las mujeres asuman más responsabilidades y compromisos horarios con las instituciones hospitalarias y que ayude a construir y consolidar el imaginario que destaca que los hombres son mejores líderes y más eficientes en los puestos de toma de decisiones.

Otro argumento, íntimamente relacionado con lo anterior y con importantes consecuencias sobre la promoción, es la identificación del poder con la figura del hombre y la masculinidad. Que el hombre en una organización detente el poder se considera algo normal, pero que una mujer posea un cargo de autoridad no está previsto socialmente, genera desconfianza y duda. Algunas autoras señalan que inclusive cuando se llega a considerar a una mujer como fuente de poder solamente se la reconoce como una excepción (Susi, 1998, Cabré i Pairet y Ortiz Gómez, 2001; Sartori, 1998). Finalmente, esta concepción del poder como típicamente masculino acaba por afectar a los criterios de la selección de cargos de responsabilidad.

En resumen, si partimos de que 1) las definiciones de conceptos como éxito, competencia, capacidad de tomar decisiones o liderazgo están asociadas con la figura masculina y 2) que aquellos que se encargan de seleccionar, o tienen poder sobre la selección del personal, tienden a hacerlo entre candidatos que son similares a ellos; se puede deducir que, más que evaluar los indicadores de éxito de las mujeres según los parámetros estandarizados por un discurso masculino (por ejemplo,

assessment (meta-analysis)". Am J Psychiatry. 161(12): 2295-302, y SCHERNHAMMER, E. (2005). "Taking their own lives the high rate of physician suicide". $N$ Engl J Med. 352(24): 2473-6. 
analizar cuánto publican las mujeres u observar qué tipo de gestión hacen), e intentar que asuman prácticas según ese estándar, lo que hay que cambiar es el criterio de lo que se considera exitoso en la promoción profesional.

En el estudio de Delgado Sánchez et al., las médicas declararon que su entorno profesional no les otorga suficiente autoridad. "Por un lado, creen que ciertas cualidades, como la rapidez o la fortaleza física, consideradas socialmente masculinas, y de las que ellas supuestamente carecen, son necesarias en algunas ocasiones para recibir autoridad de los pacientes. Y, por otro, perciben que los valores que se atribuyen a las mujeres en el sistema de género no están cargados, simbólicamente, de autoridad" (2003; 603). Se podría derivar de estos estudios que, si bien a los hombres se les otorga un cheque de confianza al asumir un cargo de toma de decisiones (se considera de antemano que "lo harán bien"), a las mujeres les pasa todo lo contrario, lo que provoca mayor estrés, obstaculiza su función, y les obliga a demostrar con más ímpetu sus capacidades de gestión bajo la mirada atenta de sus compañeros. Muchas veces esta presión desincentiva a las mujeres para fortalecer su trayectoria jerárquica. Otro factor institucional que se suele poner como causante de la falta de promoción entre las mujeres es la carga horaria que se exige, sobre todo en el sector privado. Según Reed et al. (2007), en algunas instituciones la exigencia horaria es de 60 y 70 horas semanales, marcando un perfil claro: personas jóvenes, sin responsabilidades y que, por lo general, son hombres (Reed et al. 2007, Cortés-Flores et al. 2005).

\section{Caracterizando a los médicos y a las médicas: entre las diferencias y las desigualdades.}

Para realizar la cuantificación y caracterización de la participación de mujeres y hombres en el sector sanitario se han tomado y analizado diferentes fuentes de datos estadísticos. Así, se han analizado aquellos datos secundarios que nos dan información sobre el colectivo de médicos y médicas. Las fuentes analizadas son los datos de la Encuesta de Población Activa (EPA), de la Estadística de Establecimientos Sanitarios con Régimen de Internado (EESRI) y de las pruebas de acceso a las plazas de Médico Interno Residente (MIR). Estas tres fuentes de información resultan complementarias ya que cada una de ellas da información diferente.

El análisis de la Encuesta de Población Activa EPA del 2006 nos permite una aproximación a las características estructurales del colectivo de médicos y médicas. Se ha realizado un análisis bivariado con tablas de contingencia y las variables que se han considerado relevantes son edad, relación laboral, empleo público y privado, tipo de contrato y jornada de trabajo, desagregando por sexo. La muestra del primer trimestre del 2006 se compone de 620 personas, 353 hombres y 267 mujeres $(43 \%)$. La introducción de la variable edad muestra diferencias importantes entre los distintos grupos de edad. La edad media para el conjunto de la muestra es de 44,7 años (47,8 para los hombres y 40,7 para las mujeres). La pirámide de población nos muestra distintas distribuciones para hombres y mujeres. La franja de edad con 
mayor representación es, en los dos casos, la que comprende las edades de entre 45 y 49 años. Por encima de esa edad hay mayor presencia de hombres y, por debajo, mayor presencia de mujeres.

La proporción entre hombres y mujeres da lugar al cálculo de los ratios de feminidad y masculinidad, para los distintos grupos de edad observados. Destaca el comportamiento opuesto que tiene un sexo respecto al otro. Los ratios de feminidad empiezan en sus niveles más altos en las edades más jóvenes y van descendiendo situándose por debajo del 1 a partir de los 45 años. También encontramos diferencias en el estado civil de los individuos que componen la muestra: entre los hombres el porcentaje de casados es significativamente mayor $(84,5 \%)$ que entre las mujeres $(63,7 \%)$. Hay que tener en cuenta el efecto de la estructura por sexo y edad antes comentada: la mayor concentración de mujeres en las edades más jóvenes, en un contexto social donde se ha retrasado la edad media para el matrimonio y la maternidad, hace incrementar el peso de mujeres solteras en relación a los hombres, mayoritariamente concentrados en edades más maduras. Los porcentajes de personas separadas o divorciadas se mantienen igual para ambos sexos.

En relación a las características de la ocupación podemos empezar apuntando que el empleo en el sector sanitario está claramente determinado por el empleo público $(67,50 \%$ del empleo principal es asalariado del sector público). En la distinción por sexo, observamos que entre las mujeres el porcentaje de asalariadas del sector público sube hasta un $72 \%$, mientras que para los hombres es de $64,3 \%$. La menor presencia en el sector público entre los hombres se explica por un mayor peso de empresarios con asalariados (12\%), situación que entre los hombres ocurre más del doble de veces que entre las mujeres. Esta mayor concentración, sin embargo, no nos permite confirmar la existencia de relación entre sexo y situación profesional ${ }^{4}$.

Uno de los elementos clave para entender si hay o no discriminación en el mercado de trabajo es el tipo de relación laboral que se establece. La calidad de los puestos de trabajo analizados tiene un dato importante en el carácter permanente o temporal del contrato de trabajo. Aquí sí se observan diferencias estadísticamente significativas entre hombres y mujeres: el porcentaje de precarización de los hombres es de un $18 \%$, en cambio el de las mujeres es de un $37,8 \%$. Esta diferencia se matiza en función del grupo de edad al que nos referimos. En los grupos de edad hasta los 40 años las mujeres presentan porcentajes de temporalidad superiores a los de los hombres, excepto en el grupo de menos de 25 años. Es interesante señalar que mientras que para los hombres la evolución al contrato indefinido es más rápida y clara, para las mujeres es más prolongada: en el grupo de edad de 40 a 44 años,

\footnotetext{
${ }^{4}$ Esto se corrobora con el Test Chi-cuadrado, que refleja una probabilidad de cometer el Error Tipo I de 0,068. Por tanto la conclusión es que no puede rechazarse la hipótesis nula de inexistencia de asociación. El sexo, por consiguiente, no condicionaría la situación profesional del colectivo.
} 
prácticamente un $85 \%$ de los hombres gozan de contrato indefinido, mientras que las mujeres igualan este porcentaje en el grupo de edad de 45 a 49 años. Las mujeres han de esperar hasta los 45 años para equipararse en porcentaje a los hombres respecto al contrato indefinido.

Otra dimensión que describe la situación en el empleo es el tipo de jornada laboral en la que se trabaja: sólo el $11 \%$ de la muestra tiene una jornada parcial, y éste es más elevado entre mujeres que entre hombres. Un último aspecto a comentar sobre los datos de ocupación de la EPA es la situación de pluriempleo. En la muestra estudiada, un poco más del $20 \%$ declara tener un segundo empleo. Las diferencias entre ambos sexos son en este caso significativas. Entre los hombres más del $25 \%$ tiene un segundo empleo.

El análisis de la Encuesta de Hospitales del Ministerio de Sanidad (del año 2003) nos permite tanto un análisis de datos generales como por especialidad. De la matriz "personal hospitales 2003" $(\mathrm{n}=15.715$, donde: médicos $\mathrm{n}=10.334$ y médicas $\mathrm{n}=$ 5.381) se ha llevado a cabo un análisis descriptivo mediante tablas de frecuencias en relación a las variables sexo y jornada laboral. Primero con todos los casos de la matriz y en una segunda fase filtrando la totalidad por la variable especialidad (servicios médico y quirúrgico, otros servicios sanitarios, personal no médico).

Las distribuciones porcentuales de los profesionales sanitarios por razón de sexo están claramente diferenciadas según el tipo de servicio en el que se encuentran. Si bien hay servicios médicos en los cuales aún siguen siendo más numerosos los hombres (como por ejemplo en traumatología) encontramos en otros una presencia más igualitaria de médicas (como en los servicios de pediatría, psiquiatría, medicina general). Sin embargo en los servicios quirúrgicos, englobados en la única categoría de "cirugía", la proporción de los profesionales según su sexo refleja una realidad casi de feudo profesional ya que encontramos un $81 \%$ de hombres y un $19 \%$ de mujeres.

Entre el personal no médico predominan las mujeres, y en las únicas categorías en las que hay mayoría de hombres son dirección-gestión (52,5\%), por oficio cualificado $(76 \%)$ y otros profesionales no sanitarios $(60 \%)$. Hay que tener en cuenta que el resto de unidades o servicios a los que estamos haciendo referencia están considerados socialmente como profesiones femeninas, como por ejemplo enfermería, fisioterapia, auxiliares de clínica, ayudantes sanitarios, administrativos, matronas, asistentes sociales, etc.

En relación a la jornada laboral (diferenciada en dos categorías: $>36 \mathrm{~h} / \mathrm{sem}$ o $<36 \mathrm{~h} / \mathrm{sem}$ ) se observa una misma tendencia por razón de sexo. Es decir, la distribución de realización de la jornada de $>36 \mathrm{~h} / \mathrm{sem}$ y la de $<36 \mathrm{~h} / \mathrm{sem}$ es igual para médicos y médicas (60\% hombres y $40 \%$ mujeres). Esta situación se invierte en las especialidades de psiquiatría $(53 \%$ de médicas hacen jornada $<36 \mathrm{~h} / \mathrm{sem})$, se iguala en pediatría $(53 \%$ hombres y $47 \%$ mujeres con jornada $<36 \mathrm{~h} / \mathrm{sem})$ y ginecología $(52 \%$ hombres y $48 \%$ mujeres con jornada $<36 \mathrm{~h} / \mathrm{sem})$. Dentro del ámbito de otros servicios sanitarios, observamos que hay más mujeres $(53,5 \%)$ en los servicios centrales que realizan la jornada $>36 \mathrm{~h} / \mathrm{sem}$. 
Por otra parte se ha realizado el análisis de los datos del examen de Médico Interno Residente (MIR) entre 1988 y 2005. Este análisis nos permite conocer los resultados (con información acerca del orden obtenido en la prueba, el origen geográfico del candidato, su sexo, la universidad de procedencia así como el centro y la especialidad médica elegido) de las pruebas de acceso a las plazas de médico residente realizadas entre 1988 y $2005^{5}$. Con las variables que contiene esta base de datos, todas ellas de carácter cualitativo, se pueden analizar dos cuestiones primordiales: 1) el comportamiento que tienen hombres y mujeres a la hora de elegir el centro de destino en relación con su origen geográfico y 2) cómo influye el sexo del candidato en la especialidad médica escogida. En este segundo apartado debería también considerarse el número de orden conseguido en las pruebas MIR. Sin embargo, una representación gráfica de la distribución de notas entre hombres y mujeres permite observar que, con pequeñas salvedades, no hay diferencias significativas.

Para las variables 'origen geográfico' y 'centro de destino en las pruebas MIR' y a lo largo de todo el período estudiado, las dinámicas de comportamiento entre hombres y mujeres son similares. Ambos prefieren estudiar la carrera de medicina lo más cerca posible de su domicilio y ambos también eligen un centro de destino lo más cerca posible de su domicilio o, de forma residual, del lugar donde han realizado sus estudios. Excepcionalmente hay frecuentes desplazamientos que tienen como destino los centros de Madrid y Barcelona.

Para las variables 'sexo' y 'especialidades médicas en las pruebas MIR' estudiamos la distribución por sexo en la elección de especialidad en el ámbito de la sanidad. Para ello hemos utilizado los resultados de los exámenes de acceso a las plazas de MIR de distintos años: 1988, 1989 y 1990, por una parte, y 2003, 2004 y 2005. La limitada y poco fiable información de los años noventa nos ha hecho desestimar su uso. A grandes rasgos, el análisis realizado muestra que el sexo del candidato a MIR no es un elemento neutral en la elección de la especialidad médica en la cual ese titulado o titulada quiere formarse. Esto es, existen especialidades médicas feminizadas y otras masculinizadas. Para analizar la información se han agrupado las distintas especialidades médicas: 1) Medicina especial; incluye las especialidades de medicina específica de áreas profesionales, grupos poblacionales concretos etc. 2) Cirugías; incluye las diferentes especialidades relativas a la cirugía, con excepción de la neurocirugía. 3) Neurologías; formado por las especialidades de Neurología, Neurofisiología clínica y Neurocirugía. 4) Aparatos y órganos; incluye todas aquellas especialidades médicas vinculadas a un aparato, órgano o tejido del cuerpo. 5) Conducta; incluye las especialidades de Psiquiatría y Psicolo-

${ }^{5}$ Los datos relativos al periodo comprendido entre 1995 y 1997 son de mala calidad, en tanto que contienen escasa dispersión en relación a las especialidades escogidas junto con un elevadísimo número de datos perdidos, lo que hace desestimar su utilización por falta de representatividad estadística. 
gía clínica. 6) Otras especialidades, donde se han asignado todas las especialidades que no se podían incluir en ninguno de los grupos precedentes.

Existen algunos tipos de especialidad médica claramente dominados por el sexo masculino. En particular, la cirugía y las especialidades vinculadas a la neurología ( 8 de cada 10 candidatos a MIR, con plaza adjudicada o en reserva, que escogieron cirugía eran hombres). Otros grupos de especialidad sujetos al predominio masculino son, en este caso, las neurologías y las relacionas con aparatos u órganos. Las medicinas especiales, donde se halla la Medicina familiar y comunitaria y las especialidades de conducta presentan una mayoría de mujeres, aunque en estas se está en una situación más próxima a la paridad y, por lo tanto, con una ratio más parecida a la distribución general de la población.

Este patrón de género en las especialidades médicas, sin embargo, no se mantiene a lo largo del tiempo en todos los casos. Así, los grupos de especialidad Aparatos y Órganos y Neurologías, que en los últimos años de la década de los 90 se distinguen por ser fundamentalmente profesiones de hombres, invierten su carácter a partir del 2000. Las cirugías, especialidades claramente marcadas por la presencia de hombres en el inicio de nuestro corte temporal, convergen hacia la paridad de sexo, con especial intensidad en el último año de análisis. El grupo Medicina especial, que en los primeros años muestra un equilibrio en la presencia de hombres y mujeres consolida un cambio hacia el género femenino a lo largo del periodo estudiado. Asimismo, las especialidades de Conducta aparecen en el primer lustro de 2000 como una rama médica claramente femenina, tras el cambio de tendencia producido a finales de los ochenta y primeros noventa. Podemos afirmar, pues, que género y elección de especialidad en medicina no son elementos independientes.

\section{Discursos que legitiman: lo que dicen las entrevistas}

El trabajo empírico realizado en la investigación que da pie a este artículo, ha consistido en realizar entrevistas en profundidad a personal médico de tres hospitales ${ }^{6}$. Se realizaron 72 entrevistas en profundidad a médicas y médicos adjuntos de diferentes especialidades, médicas y médicos con cargos de gestión (jefes de servicios), personal de recursos humanos y otro tipo de informantes claves que tienen

\footnotetext{
${ }^{6}$ Esta fase del trabajo de campo requirió una decisión metodológica importante: estudiar únicamente hospitales y no Centros de Atención Primaria. Se optó por no estudiar esas unidades del sistema sanitario porque, una vez hechas las entrevistas exploratorias, se constató que en esas unidades el desarrollo de trayectorias profesionales era de muy pocas posibilidades de promoción. La selección de los hospitales estudiados se hizo entre hospitales grandes, según número de camas, y en base a la tipología que resulta según la propiedad y gestión del centro. Se diferencian 3 tipos: centros de propiedad pública y gestión pública, centros de propiedad pública y gestión privada, centro de propiedad y gestión privada.
} 
una experiencia relevante en materia de promoción y trayectorias médicas profesionales. Hemos articulado las ideas obtenidas en las entrevistas alrededor de varios ejes que sostienen la estructura de las percepciones e interpretaciones de médicos y médicas.

\subsection{Sobre las formas de inserción, de promoción y los perfiles profesionales}

Es posible encontrar tres perfiles típicos entre el personal médico: el asistencial, el investigador-docente y el gestor. Debido a que la estructura hospitalaria es relativamente "plana", la carrera jerárquica genera pocas oportunidades para las trayectorias profesionales y la competencia, por tanto, es muy alta. Además, cuanto más alto es el cargo, menos lugar ocupan las tareas asistenciales. Esto repercute sobre los estímulos del personal médico para acceder a cargos de responsabilidad. Agregado a estos obstáculos comunes a todos, las mujeres se enfrentan con otras limitaciones debidas a su género.

En sistemas de selección donde no se elige por mérito sino por la confianza, las redes sociales y los "lobbys", las mujeres tienen menos recursos ya que éstas tienden a participar menos que ellos. Paralelamente, esto les genera menos alicientes para acceder a cargos de responsabilidad. Finalmente, todas estas razones parecen impactar sobre las identidades de las mujeres. Ellas parecen "elegir" más los perfiles asistenciales, los hombres en cambio, parecen elegir éstos últimos perfiles investigador-docente y gestor. Esto explica porqué muchas mujeres dicen que ellas no tienen "interés" en promocionar o crecer dentro de la estructura de decisiones de un hospital. Lo que en principio aparece como elección personal, puede tener un trasfondo de constreñimiento social ("no tengo más opciones").

El modelo de gestión empresarial que se ha impuesto sobre los hospitales es reciente $^{7}$, y por tanto, las ofertas de formación en materia de gestión y administración de los recursos sanitarios son bastantes nuevas. Debido a la novedad de los cursos y los protocolos de formación (en donde todas las personas pueden adquirir las mismas habilidades y demostrarlas a través de títulos oficiales), sumado a que las mujeres han sido socializadas profesionalmente en un perfil más "asistencialista", el grueso del personal médico que actualmente accede a cargos de gestión ha sido elegido por criterios que se denuncian como "masculinizados".

Hay que aclarar que las formas de discriminación son tácitas y difíciles de detectar, inclusive para las propias mujeres. Es difícil encontrar relaciones de subordinación con pautas explícitas o formales en contextos sociales democráticos. Amén de que las instituciones hospitalarias no son organizadas con criterios democráticos ni tienen como función la distribución del poder de manera equitativa, la discriminación directa no se puede producir debido a que las relaciones sociales no pueden ser

${ }^{7}$ Para un análisis de las nuevas formas de gestión de los servicios sanitarios se puede consultar Pastor (2006) y para profundizar en la reestructuración de la profesión médica, ver Irigoyen (2011). 
legitimadas en base a pautas expresamente autoritarias. Esto hace más difícil la detección de las causas de la inequidad de género.

\subsection{Sobre la naturalización y la interiorización de las características socia- les del género}

Un argumento común que han desarrollado los entrevistados es que las mujeres tienen ciertas cualidades y hábitos que impiden el pleno desarrollo laboral. El hecho de ser mujeres y la asociación "acrítica" del sexo con el rol tradicional dentro de la familia es otra de las variables más nombradas para explicar la desigualdad en la distribución del poder dentro de los hospitales.

Casi todas las personas entrevistadas, en algún momento de la entrevista, argumentan que la incompatibilidad de la vida familiar con la vida laboral es una barrera que impide a las mujeres acceder a cargos de gestión y responsabilidad. La carga horaria, los conflictos, el aumento de responsabilidades hacen que los hombres, sean más competitivos para alcanzar los puestos de mando intermedio. Detrás de esto se trasluce la concepción acerca de los roles familiares; los hombres no se abocan a los cuidados de la pareja, el hogar y/o los hijos y así pueden dedicarle más horas y atención al empleo. En pocos casos se cuestiona el rol de la mujer y el hombre en la vida privada. Pocas personas no esperan de las mujeres que sean madres, esposas, amas de casa, además de médicas, en cambio los hombres en el mundo hospitalario son considerados únicamente en su rol de médicos. Este discurso impacta sobre las identidades de las profesionales. Hay muchas mujeres médicas que niegan la desigualdad, prefieren el perfil asistencial y declaran no estar interesadas en la promoción jerárquica "porque están cómodas en el lugar que están".

En otras palabras, el determinante cultural o el "discurso hegemónico" por el cuál la mujer debe atender primero las demandas de una vida familiar o privada y, luego, compatibilizarla con el trabajo impacta sobre los estímulos de las médicas para acceder a los puestos de gestión. En algunos casos, los fracasos previos convencen a las médicas del argumento de la compatibilidad familiar y las sobre exigencias del cargo. Es probable que como no han tenido éxito en el acceso a cargos de mando, busquen motivos para reforzar su autoestima y justificar su perfil profesional. Agregado a ello, el discurso masculino hegemónico le facilita explicaciones y herramientas simbólicas para menguar los efectos negativos de estas frustraciones y no buscar otro tipo de respuestas o acciones con más altos costos emocionales.

Esta estigmatización también funciona como criterio de exclusión de aquellas personas que influyen en la selección del personal. Más allá de que objetivamente un cargo de gestión exija más horas y más compromiso que un puesto de personal médico adjunto, y que muchas mujeres no estén dispuestas a aceptarlo, esto funciona como argumento que explica de antemano porqué las médicas no promocionan jerárquicamente al igual que sus pares hombres. En otras palabras, este discurso opera como un argumento que legitima la discriminación de género a la hora de seleccionar a aquellas personas que van a promocionar.

Las anteriores líneas dan paso a la siguiente reflexión: para enfrentar las formas de discriminación es necesario reglar los criterios de selección e implementar, hacia 
el interior de la carrera profesional sanitaria, las leyes ya existentes de compatibilidad entre la vida privada y laboral. Pero también es imprescindible (y tal vez lo más difícil) generar políticas públicas que permitan fundar procesos de socialización alternativa, aprendizaje y autorreflexión, para que las propias mujeres puedan detectar situaciones de discriminación y que permitan, también, la modificación de los roles masculinos y femeninos dentro y fuera de la vida privada.

\subsection{Sobre el capital social y las dinámicas intra institucionales}

A través de las entrevistas se puede observar que la confianza entre quien selecciona y la persona seleccionada, es un elemento crucial a la hora de acceder a un cargo de responsabilidad. Las relaciones que se tengan con las compañeras y los compañeros de trabajo, pero sobre todo con las personas que toman decisiones dentro del hospital (gerencia, dirección médica, jefatura de servicios) son fundamentales a la hora de promocionar. Por otro lado, también se transluce en las entrevistas a informantes claves que muchas médicas son poco reflexivas frente a su situación de desigualdad en el acceso a cargos de gestión y responsabilidad.

En otras palabras, las redes masculinas son preponderantes dentro de los hospitales y son una de las variables explicativas de las desigualdades de género en el acceso a cargos de poder. No obstante, se puede observar que las mujeres han creado su propio capital para contrarrestar a las primeras. Una política activa dirigida a romper con la segregación por sexos en estas redes o a fortalecer las alianzas femeninas podría ayudar a desatar el nudo de la inequidad.

\section{Conclusiones}

La primera idea expuesta en este artículo es que, a pesar de la progresiva feminización de la comunidad médica, la presencia de mujeres entre el colectivo de personal médico de los hospitales continúa siendo notablemente inferior a la de los hombres, especialmente en determinadas especialidades, por ejemplo la cirugía o en determinados niveles profesionales, como por ejemplo las jefaturas de servicios o las direcciones de los hospitales. Esta segregación horizontal y vertical rompe con la imagen de la carrera médica como un ámbito igualitario en el acceso y la promoción profesional de los hombres y de las mujeres.

Los principales problemas detectados a la hora de obtener la información son las falsas creencias y los estereotipos sostenidos por el discurso hegemónico. Este discurso dominante niega la existencia de discriminaciones hacia las mujeres en el ámbito sanitario y se encubre por el mito de que dichas organizaciones se presentan a sí mismas como meritocráticas. La primera dificultad encontrada es, pues, la negación, por parte de los y las propias protagonistas, de nuestro objeto de estudio: la existencia de discriminaciones sexistas. La mayoría del personal médico -casi todos los hombres y muchas mujeres-, consideran que las organizaciones sanitarias no son sexistas ni tienen ningún tipo de influencia patriarcal.

Así pues, pese a las diferencias encontradas en los discursos de hombres y muje- 
res, cabe destacar la posición unánime que mantienen los médicos y las médicas respecto a la no existencia de discriminación en el seno de los hospitales. Es importante hacer mención, cuando se habla de discriminaciones, a que, en prácticamente todos los casos, cuando se habla de discriminación ésta se identifica con discriminaciones directas, obviando, por desconocimiento o interés, otras formas de discriminación indirectas, que son las que efectivamente se dan. Es más, se pueden distinguir, siguiendo el análisis que hace Arranz (2004) para las dinámicas universitarias, tres posiciones en la explicación de la asimetría o la discriminación de género, nunca considerada sexismo:

(1) Justificaciones esencialistas que reconocen la existencia de discriminaciones en el contexto sanitario-hospitalario: las desigualdades son el resultado de las diferencias biológicas, psicológicas o de socialización entre los hombres y las mujeres, dando lugar a roles diferenciados. La desigual distribución en los diferentes campos de especialización o en la carrera profesional es fruto de que las mujeres privilegian sus roles femeninos, como por ejemplo el deseo de ser madre, sobre objetivos profesionales más ambiciosos. Según este punto de vista las mujeres escogerían "libremente" esta situación.

(2) También aparece otro tipo de argumento más igualitario que, a diferencia del anterior, no considera significativas las diferencias de género observadas en la vida hospitalaria. Las desigualdades acabarán desapareciendo per se, con el tiempo; superarlas es una cuestión generacional, y se hace referencia a la lentitud que implica toda transformación social. La solución, en este caso, pasa por saber esperar.

(3) Justificaciones meritocráticas, que se aferran a la neutralidad y objetividad propia de los procesos de selección del personal.

En ninguna de estas representaciones del colectivo médico se reconoce la existencia de formas de dominación masculina como principios estructuradores de la realidad. Es más, se niega la existencia de conflicto y se intenta cubrir poniendo por ejemplo la feminización que presentan determinadas especialidades (segregación horizontal) como una muestra del entorno igualitario que representa la profesión médica. Estas representaciones ideológicas calan en las prácticas médicas (tanto con respecto a las instituciones como al ejercicio de la profesión) de tal forma que (estas pre-nociones o racionalizaciones) protegen la cultura "masculina" de la profesión para seguir dominante en aras de la racionalidad, la neutralidad y la objetividad.

Con este artículo se ha constatado también que, si bien las médicas, en su mayor parte, se auto-reconocen como un colectivo con la misma valía que los hombres (dedicación, méritos, formación, etc.), al mismo tiempo declaran encontrarse ante una situación social y profesional más "desfavorable" que ellos (por ejemplo, en los apoyos recibidos por parte de los jefes, en las dificultades relacionadas con la maternidad, etc.). La explicación pasa por entender la forma en que se establecen las relaciones de género dentro las organizaciones. En síntesis, podemos identificar una serie de obstáculos que las mujeres profesionales deben combatir a lo largo de 
su trayectoria profesional. Los resumimos en tres:

(1) limitaciones derivadas del protagonismo femenino en la asunción de responsabilidades respecto al trabajo reproductivo,

(2) dificultades asentadas en la socialización de género y

(3) problemas relativos al dominio masculino sobre la práctica médica y las redes sociales en las que esa práctica está insertada.

Se ha mostrado que la práctica médica no es ajena a la discriminación por razón de género existente a la sociedad, sino que contrariamente a la visión generalizada, es un espejo. No obstante, la mayoría de sus miembros no tienen esta percepción, ni tampoco las mujeres, especialmente las más jóvenes. Sólo en el caso de aquellas que se plantean promocionar $\mathrm{y}$, sobre todo, si quieren formar una familia, perciben las primeras dificultades. A partir de los testimonios recogidos se ha observado que, bajo la apariencia de un sistema de promoción profesional formalmente igualitario, basado en las calificaciones (méritos) y la experiencia, aparecen unas limitaciones formales e informales en el desarrollo de la carrera profesional, que configuran las expectativas y los horizontes profesionales de los médicos y las médicas.

\section{Referencias bibliográficas}

Anderson, B., Zinsser, J. (1991). Historia de las mujeres. Una historia propia. Barcelona: Crítica.

Arranz, F. (2000). Las políticas públicas a favor de las mujeres, Madrid: Instituto de Investigaciones Feministas de la Universidad Complutense de Madrid.

Arranz, F. (2004). Las mujeres y la universidad española: estructuras de dominación y disposiciones feminizadas en el profesorado universitario, Política y sociedad, Vol. 41, 2: 223-242.

Arrizabalaga, Pilar y Valls Llobet, Carme (2005). Las mujeres médicas: de la incorporación a la discriminación. Medicina Clínica, Barcelona; 125(3): 103-7.

Barbera, E; Sarrió, M; Ramos, A. (Coord.) (2000). Mujeres directivas: promoción profesional en España y el Reino Unido. Valencia: Estudi Universitari d'Estudis de la Dona. Universitat de València. Col·lecció Quaderns feministes.

Bourdieu, P. (2000). La dominación masculina. Barcelona: Anagrama.

Burín, M. (1996). Género, psicoanálisis, subjetividad. Buenos Aires: Paidós. 
Cabré i Pairet, M; Ortiz Gómez, T. (Ed.) (2001). Sanadoras, matronas y médicas en Europa, siglos XII-XX. Barcelona: Icaria.

Camps, B. (2009). Informe sobre demografia col-legial 2009. Informe Anual 2009 Barcelona: Col-legi Oficial de Metges de Barcelona. Pág. 18-19.

Castaño Collado, C. (investigador principal) (2009). Mujeres y poder empresarial en España. Madrid: Instituto de la Mujer. Colección Estudios, 108.

Colomer Revuelta C.; Peiró Pérez, R. (2002). ¿Techos de cristal y escaleras resbaladizas? Desigualdades de género y estrategias de cambio. Gaceta Sanitaria 16(4): 358-60.

Cortés-Flores, A.; Fuentes-Orozco, C.; López Ramírez, M. K. L.; Velázquez Ramírez, G.A.; Farias-Llamas, O. A.; Olivares Becerra, J. J.; González-Ojeda, A; (2005). Medicina académica y género. La mujer en especialidades quirúrgicas. Gaceta Médica Mexicana, México; Vol.141 No. 4: 341-344.

Delgado Sánchez, A.; Távora Ribero, A.; Ortiz, T. (2003). Las médicas, sus prácticas y el dilema de la feminidad. Estudios de sociolingüística, Universidad de Vigo, 4 (2).

Dodd, D. E.; Gorham, D. (1994). Caring and Curing: Historical Perspectives on Women and Healing in Canada, Otawa: University of Ottawa Press.

Elston, M. A. (2001). Run by women, (mainly) for women: medical women's hospitals in Britain, 1866-1948. Clio Medica, 61: 73-107.

Ely, R.; Meyerson, D. (2000). Theories of gender in organizations: a new approach to organizational analysis and change. Research in Organizational Behaviour, Volume 22: 103-151.

Ely, R.; Padavic, I. (2007). A feminist analysis of organizational research on sex differences. Academy of Management Review, 32(4): 1121-1143.

García de Cortázar, M. L.; García de León Álvarez, M. A. (1996). Las catedráticas de Universidad. Un caso de mujeres en minoría en un círculo de poder androcéntrico. En Brullet, C.; Carrasquer, P. (comp.). Sociología de las relaciones de género. Congreso de Sociología, Granada 1996. Madrid: Instituto de la Mujer-Ministerio de Trabajo y Asuntos Sociales. Pág. 179-188.

Gil Deza, E. (2005). El síndrome burnout en la atención clínica, conferencia realizada en las Jornadas Intramed, México, 1.06.2005. 
INE (2010). Mujeres y hombres en España 2010. Madrid: Instituto Nacional de Estadística.

Irigoyen, J. (2011). La reestructuración de la profesión médica. Política y Sociedad, 48 (2): $277-293$.

Maruani, M., Rogerat, C., Torns, T. (dirs.) (2000). Las nuevas fronteras de la desigualdad. Hombres y mujeres en el mercado de trabajo. Barcelona: Icaria.

Miller, J.B (1987). Hacia una nueva psicología de la mujer. Barcelona: Paidós.

Miles, A. (1991). Women, health and medicine. Philadelphia: Oxford University Press.

Nicolson, P. (1997). Poder, género y organizaciones. ¿Se valora a la mujer en la empresa?. Madrid: Ed. Narcea. Colección mujeres.

Ortíz Gómez, T. (2007). "La práctica Sanitaria en la historia. ¿Una cuestión femenina?”. Eidon, 2007, Pág. 61-65

Ortiz Gómez, T.; Delgado Sánchez, A.; Távora, A. (1999). Trabajo, género y medicina. Actividad profesional de las médicas españolas en la segunda mitad del siglo XX. Madrid: MTAS.

Ortíz Gómez, T.; Birriel-Salcedob, J.; Ortega del Olmoc, R. (2004). Género, profesiones sanitarias y salud pública. Gaceta Sanitaria. ; 18, Supl. 1:189-94.

Pastor, I. (investigadora principal) (2009). Mujeres en Sanidad: trayectorias profesionales y acceso a cargos de responsabilidad, Instituto de la Mujer, Madrid.

Pastor, I. (2006). ¿La gestión privada en la Administración pública? El caso de los servicios sanitarios hospitalarios en Cataluña. Madrid: Consejo Económico y Social.

Recio, A.; Recio, C. (2005). Les desigualtats en el mercat de treball. Panel de les desigualtats socials a Catalunya. Barcelona: Fundació Jaume Bofill.

Reed, V.; Buddeberg-Fischer, B. (2001). Career obstacles for women in medicine: an overview. Medical Education, 2001; 35 (2): 139-147.

Riska, E; Wegar, K. (eds.) (1993). Gender, work and medicine. Women and the medical division of labour, London: Sage. 
Sánchez, C. (2003a). Ciudadanía y Derechos Humanos. Una mirada desde el género. En P. Pérez Cantó (coord.), Mujeres de dos mundos: ciudadanía social de las mujeres latinoamericanas. Madrid: Comunidad de Madrid.

Sánchez, C. (2003b). Genealogía de la vindicación. Beltran, E.; Maquieira, V. (eds.). Feminismos: Debates teóricos contemporáneos. Madrid: Alianza. Coleccion Ciencias Sociales.

Sartori, D. (1998). La autoridad en cuestión. En HIPATIA. Autoridad científica, autoridad femenina. Madrid: HORAS y HORAS, Pág. 59-85.

Schroen, A; Browstein, M; sheldon, G. (2004). Women in Academic General Surgery, Academic Medicine, 79: 310-318.

Susi, E. (1998). Las felices anomalías. En HIPATIA. Autoridad científica, autoridad femenina. Madrid: HORAS y HORAS, Pág. 13-20.

Themis, Asociación de Mujeres Juristas (2009). Las mujeres y la toma de decisión. Informe 2009.

Torns, T., Carrasquer, P., Parella, S., Recio, A. (2007). Les dones i el treball a Catalunya: mites $i$ certeses. Barcelona: Generalitat de Catalunya. Institut Català de les Dones.

Torns, T. (1999). Las asalariadas: un mercado con género. En Miguélez, F.; Prieto, C. Las relaciones de empleo en España. Madrid: Siglo XXI.

Ulstad, V.K. (1993). How women are changing medicine. Journal of the American Medical Women's Association, 48(3): 75-78.

Valcarcel, A. (1994). Sexo y filosofia. Sobre la "mujer" y el "poder". Barcelona: Ed. Anthropos. 